\title{
RESUMEN.
}

Con la globalización de los mercados, las empresas han expandido su ámbito de actuación, lo cual hace que el interés por sus actividades traspase las fronteras nacionales. El actual escenario de las organizaciones, obliga a éstas a adaptar sus sistemas de información y comunicación con nuevas tecnologías, ya que se entiende que ellas, puestas al servicio de la información, favorecen las decisiones obteniendo ventajas competitivas. Por esto, es que nace la necesidad de crear un lenguaje común para la transmisión de información financiera y empresarial. En Chile con la adopción de las Normas Internacionales de Contabilidad, la Superintendencia de Valores y Seguros opta por utilizar el XBRL como base tecnológica para la estandarización de la información. A cinco años de su implementación, es importante analizar la experiencia de las empresas que han tenido que adoptar esta medida. En la investigación se aprecia que el uso del XBRL aún se encuentra en su etapa inicial. A pesar de que las empresas cumplen con la exigencia impuesta por el ente regulador, los usuarios no aprovechan los beneficios que genera la estandarización de la información, optando por otros reportes generados internamente.

Palabras Claves: Convergencia IFRS, Formato XBRL, TICs, Reportes Financieros.

\section{ANÁLISIS DE LA EXPERIENCIA DE LAS ORGANIZACIONES, OBLIGADAS A REPORTAR A LA SUPERINTENDENCIA DE VALORES Y SEGUROS, EN LA IMPLEMENTACIÓN Y USO DEL XBRL.}

Carolina Francisca Moyano Oyanedel.

ANALYSIS OF THE EXPERIENCE OF COMPANIES, WHICH ARE FORCED TO REPORT TO THE SUPERINTENDENCY OF SECURITIES AND INSURANCE, THE IMPLEMENTATION AND USE OF XBRL.

\begin{abstract}
.
Along with market globalization companies have expanded their scope of action causing the interest for their activities to cross national borders. The current scenery of these organizations makes them adapt their information and communication systems to new technologies which favor their decision making and thus obtain competitive advantages. For this reason the necessity, to create a common language to transmit financial and entrepreneurial information, became essential. When Chile adopted the International Standards of Accountability, the Superintendency of Securities and Insurance chose to use the XBRL as the technological base for the standardization of information. Five years after its implementation, it is important to analyze the experience of the companies which had adopted this measure. In this research, it can be seen that the use of XBRL is still in its initial stage because although companies meet the specifications demanded by the regulatory entity, users do not take advantage of the benefits that the standardization of information provides. They still prefer to choose other internal reports.
\end{abstract}

Key Words: Convergence IFRS, XBRL format, TICs, Financial reports.

1 Alumna Tesista de la Carrera de Auditoría de la Universidad de Valparaíso, teniendo como profesor guía al Profesor Carlos Murat Ibaceta. 


\section{Propósito del estudio.}

Como se ha señalado, actualmente las empresas se encuentran insertas en un proceso de globalización donde es necesario crear, administrar y distribuir recursos de información, a través de redes interconectadas como lo es internet. Los avances tecnológicos han configurado un nuevo escenario digital, donde los Sistemas de Información deben ser capaces de adaptarse para proporcionar información financiera útil y oportuna a los usuarios que lo requieran, considerando que la Información es uno de los recursos más valiosos e importantes de las organizaciones. El hecho de que la información se digitalice, no quiere decir que sea compatible con todos los programas informáticos, debido a que cada organización utiliza sus propios formatos de almacenamiento de datos.

A fines del año 2006, Chile decide adoptar las Normas Internacionales de Contabilidad Financiera (IFRS), emitidas por el International Accounting Standards Board (IASB). Durante el año 2008, previo análisis, se decide adoptar el XBRL (Extensible Business Reporting Language) como base tecnológica para complementar la estandarización de la información, cumpliendo con el objetivo de hacerla comparable, transparente y de alta calidad, y que está siendo adoptado progresivamente por el mundo de los negocios. El proceso de convergencia comienza a materializarse a fines del año 2009 con la entrega de los primeros informes elaborados bajo IFRS y reportados a través de XBRL, de acuerdo a las Taxonomías elaboradas por la Superintendencia de Valores y Seguros, originando posibles ventajas y desventajas para las empresas informantes. El XBRL ha sido diseñado para simplificar la automatización e intercambio de información financiera mediante el uso del lenguaje XML, permitiendo su utilización por todos los actores que intervienen en la cadena de generar, distribuir y utilizar la información financiera.

La presente investigación tiene por finalidad analizar la experiencia en la implementación y uso del XBRL, de las organizaciones obligadas a reportar a la Superintendencia de Valores y Seguros, desarrollada a través de entrevistas a profesionales y expertos, a Diciembre de 2013. Específicamente la investigación describe la importancia del uso de las Tecnologías de Información y Comunicación en los Sistemas de Información de las organizaciones; explica la creación y evolución del XBRL a nivel mundial y local, y el uso de taxonomías para la elaboración de informes financieros. Finalmente, analiza las principales ventajas y desventajas del uso de XBRL en las organizaciones. 


\section{Teoría.}

\section{Globalización en Chile.}

La Globalización se entiende como un proceso multifacético, no solo económico, sino también cultural, político y social que tiene como expresión más determinante la interdependencia de los mercados, favorecida por las nuevas tecnologías de información y comunicación y por la liberación de dichos mercados. Además, es un fenómeno donde los acontecimientos se suceden al instante.

La globalización ha sido entendida en Chile como un proceso de liberalización total del movimiento de capitales, bienes y servicios. Además desde mediados de la década de los 70 se ha adoptado un modelo de desarrollo basado en la competencia donde se pretende que la actividad económica de la sociedad sea regulada por los mercados. Este proceso se ha visto profundizado con la firma de tratados de libre comercio, en los cuales no solo los bienes materiales son su objeto, sino también los servicios, incluyendo los servicios públicos.

Gracias al desarrollo de las exportaciones impulsadas por las relaciones comerciales con el extranjero, Chile exhibe los mejores índices macroeconómicos de América Latina y se destaca por un dinamismo económico que en muchos aspectos lo ubica entre los punteros globales del desarrollo. (Biblioteca del Congreso Nacional de Chile, 2005).

\section{Sistemas de Información y Tecnologías de Información y Comunicación (TIC).}

\section{Sistemas de Información.}

Los sistemas de Información son un conjunto integrado de procesos, principalmente formales, desarrollados en un entorno usuario-computador, que operando sobre un conjunto de datos estructurados de una organización, recopilan, procesan y distribuyen selectivamente la información necesaria para la operatividad habitual de la organización y las actividades propias de la dirección de la misma (Andreu, Ricarte, \& Valor, 1991)

\section{Tecnologías de Información y Comunicación (TIC).}

Las Tecnologías de Información y Comunicación (TIC) ocupan un lugar central en la sociedad, en la economía y al interior de las organizaciones. El concepto de TIC surge como convergencia tecnológica de la electrónica, el software y las telecomunicaciones. 
"Debemos entender por tecnología de información todo lo relacionado con la captura, transportación, proceso y uso de información, es decir, todo lo que involucra equipos computacionales (hardware), programas de cómputo de infraestructura, aplicativos (software) y las telecomunicaciones" (Bastias, 2009; p. 5)

Estas herramientas son utilizadas masivamente, no solo porque hacen posible la disminución de los costos, sino también por su impacto positivo en la información requerida en los procesos de toma de decisiones.

\section{La importancia de Internet.}

Dentro de las TIC, internet se ha transformado en una poderosa herramienta. No solo sirve para acceder a una gran cantidad de información, sino que, de manera muy significativa, permite a las organizaciones realizar comercio electrónico, relacionarse con inversionistas y proporcionar información financiera y empresarial a quienes la requieran. Entre las múltiples características que se atribuyen a internet, se puede destacar las siguientes: su alcance global, universalidad en el acceso, disponibilidad de 24 horas los 365 días del año, información permanentemente actualizada, comunicación bidireccional e interacción del usuario, contenido hipertextual, capacidad para realizar transacciones comerciales, capacidad para distribuir productos digitales, costo reducido y personalización de la comunicación, entre otras.

\section{Información Financiera.}

La información financiera es generada desde la contabilidad, siendo indispensable para la administración y el desarrollo de la empresa. Por lo tanto, es procesada y concentrada para uso de la gerencia y otros usuarios que requieran de ella para la toma de decisiones. La información financiera, se ha convertido en un conjunto integrado de estados financieros y notas, que sirven para expresar tanto la situación financiera y sus cambios, como el resultado de las operaciones de la empresa. Esta información junto con otros elementos de juicio, permiten a los usuarios evaluar el futuro de la empresa y tomar decisiones de carácter económico sobre la misma. La información contable-financiera constituye el principal vehículo de comunicación entre los directivos y los propietarios y otros terceros interesados en la marcha de la empresa, en particular cuando se trata de sociedades cotizadas en los mercados de valores, en las que las participaciones en la propiedad se encuentran dispersas y alejadas del poder de decisión, que queda en manos de los administradores (Cañibano, 2004). 


\section{Usuarios.}

Los Estados Financieros han sido preparados para proporcionar información útil en la toma de decisiones de naturaleza económica, satisfaciendo las necesidades de la mayoría de los usuarios. Algunos ejemplos de decisiones de índole económica son: decisiones sobre cuándo comprar, vender, o mantener una inversión de capital; evaluación de la responsabilidad de la gerencia; valorar la habilidad de la empresa para pagar y otorgar beneficios a sus empleados; estimar la solvencia de la empresa por préstamos que le otorguen; determinar políticas fiscales; establecer utilidades y dividendos para distribuir; preparar y utilizar estadísticas nacionales de ingresos y regular las actividades de la empresa (Montes, Montilla, \& Mejía, 2006)

\section{Los Estados Financieros.}

Los estados financieros forman parte del proceso de información financiera y son preparados y presentados para ser utilizados por usuarios externos de cualquier parte del mundo. Normalmente un conjunto completo de estados financieros comprende:

1. Balance General. También conocido como estado de situación financiera 0 estado de posición financiera. Muestra la estructura financiera de la entidad, es decir los activos, pasivos exigibles y su capital contable 0 patrimonio contable a una fecha determinada.

2. Estado de Resultados. Muestra los resultados operacionales de la entidad durante un periodo determinado. Se determina presentando los ingresos, costos y gastos, así como la utilidad o pérdida neta del periodo.

3. Estado de Cambios en el Patrimonio Neto. Muestra los cambios en la inversión de los propietarios.

4. Estado de Flujo de Efectivo. Presenta información acerca de la generación y utilización de recursos por actividades de operación, inversión 0 financiamiento en el periodo. Álvarez (2009).

5. Notas a los Estados Financieros. A través de las notas se puede presentar información adicional relevante respecto de determinadas partidas del balance y de la cuenta de resultados.

\section{La Información Financiera en Internet.}

El uso de Internet en las empresas se centra básicamente en las siguientes áreas: comercio electrónico, relaciones con los inversionistas, así como proporcionar información financiera y empresarial. 
La publicación de información financiera en Internet presenta numerosas ventajas, como la mayor facilidad de acceso, el incremento de la audiencia potencial, la posibilidad de divulgar datos en línea, la flexibilización de contenidos y el aumento en el volumen de información que es posible poner a disposición de los usuarios. No obstante, la comunicación en línea de la información financiera no está exenta de riesgos, puesto que se puede presentar información incompleta, contradictoria, no contrastable, dispersa e incluso es posible una sobrecarga informativa. En cualquier caso, la difusión de información financiera en la red ha de hacerse de forma ordenada, coherente, sistemática y, en definitiva, útil para el usuario.

Hay que tener presente, que para los stakeholders es más interesante una información perfectamente especificada y estandarizada que enormes cantidades de datos descargados en formatos (PDF, WORD, EXCEL, etc.) en los cuales la información contable puede presentarse de forma diferente por cada empresa, no siendo por tanto apta para ser introducida directamente en aplicaciones informáticas de análisis, y requiriendo una homogeneización adicional que hace necesaria la intervención de operadores humanos. (Lorca, Suarez, \& García, 2004; p 3 )

Por consiguiente, es preciso dar un paso más en la normalización contable y pasar de una situación en la que solo se regula el contenido de las cuentas a otra en la que la presentación también es objeto de interés.

Con la globalización de los mercados se hace preciso estandarizar la forma de enviar la información, es decir, llegar a un acuerdo sobre el significado de los datos que se transmiten. Así, debe especificarse claramente qué significa cada dato que se envía y se recibe, de forma que no haya lugar a ambigüedades. Los lenguajes que comúnmente se emplean para la transmisión de la información financiera en esta red mundial de datos, son fundamentalmente HTML (Hypertext Markup Language) y PDF (Portable Document Format).

En los últimos años estos lenguajes han generado una explosión de reportes desde internet, sin embargo ambos lenguajes han tenido ciertas limitaciones que reducen la rapidez y, en algunos casos, hacen difícil e ineficiente la búsqueda de información en internet (Valderrama, 2002). A partir del año 1998 surge un nuevo lenguaje llamado XML (extensible markup language), desarrollado para eliminar las limitaciones anteriormente mencionadas y que ha servido como base para el diseño de nuevos lenguajes de reportes y presentación de información financiera, como es el caso del XBRL. 


\section{eXtensible Markup Language (XML).}

Para poder entender XBRL, es necesario conocer previamente el lenguaje sobre el que está basado. A continuación se realizara una breve introducción del lenguaje $\mathrm{XML}$.

El estándar XML (Lenguaje Extensible de Marcas) es uno de los formatos más utilizados para el intercambio de información estructurada tanto a nivel local como a través de redes, creado por el W3C (World Wide Web Consortium).

Sus creadores lo definen como: "Un lenguaje de etiquetado extensible simple, pero estricto, que juega un papel fundamental en el intercambio de una gran variedad de datos. Es un lenguaje muy similar a HTML (lenguaje en que se escriben las páginas Web), pero su función principal es describir datos y no mostrarlos como es el caso de HTML. Las tecnologías XML son un conjunto de módulos que ofrecen servicios útiles a las demandas más frecuentes por parte de los usuarios. XML sirve para estructurar, almacenar e intercambiar información" (Zuñiga, 2007; p. 18).

El formato de etiquetas proporciona un significado adicional a la información ordinaria a intercambiar de forma que las aplicaciones informáticas que procesan la información sean capaces de entender dicho significado, estos son los llamados metadatos de las etiquetas.

\begin{tabular}{|c|c|}
\hline $\begin{array}{l}\text { Michael M. Miller } \\
8080 \text { Floppy Disk Drive } \\
\text { San Sushi, CA } 94078 \\
+1 \text { (617) } 515-1424 \\
\text { Birthdate: } 19 \text { October } 58\end{array}$ & 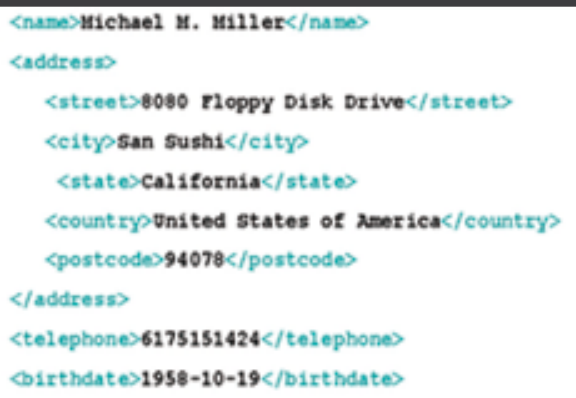 \\
\hline $\begin{array}{c}\text { Información } \\
\text { Ordinaria }\end{array}$ & $\begin{array}{l}\text { Metadatos: } \\
\text { Información acerca de la } \\
\text { información }\end{array}$ \\
\hline
\end{tabular}

Fuente: Libro Blanco 4 XBRL concepto y recomendaciones (2006).

\section{Historia de XML.}

En 1969 IBM crea el GML (Lenguaje General de Marcas) para administrar y clasificar la gran cantidad de información que poseía en esa época, para luego 
procesarla adecuadamente.

En 1986, la ISO (Organización Internacional para la Estandarización), decide estandarizar el lenguaje GML, para poder ser utilizado por quienes necesiten gestionar grandes volúmenes de información heterogénea, es así como nace el SGML (Lenguaje General de Marcas Estándar), versión mejorada con respecto a su antecesor, que proporciona un modo consistente y preciso de aplicar etiquetas para describir las partes que componen un documento, permitiendo además su intercambio entre diferentes plataformas. Sin embargo, el problema que se atribuye a SGML es su excesiva dificultad.

En 1998 aparece el lenguaje XML, derivado de SGML, pero más simplificado, eliminando las partes más engorrosas y menos útiles. XML es un metalenguaje, es decir, un lenguaje para definir lenguajes.

\section{Principales Ventajas}

Entre sus ventajas se puede destacar:

- $\quad$ Es un lenguaje fácil de leer, tanto por humanos como por máquinas.

- Es auto contenido: en un documento XML se describen los valores de los datos pero también su estructura y los nombres de sus campos.

- Gracias a su estructura jerárquica, permite representar prácticamente cualquier estructura de datos de una forma sencilla: listas, registros, árboles, etc.

- Es independiente de la plataforma tecnológica: se puede encontrar utilidades y librerías para manejar documentos XML en cualquier sistema operativo y para cualquier lenguaje de programación.

- El uso de una sintaxis estricta permite un tratamiento eficiente por herramientas adecuadas. (Morilla, 2007, p.32)

A grandes rasgos y comparando este formato con otros de gran utilización, con XML se logra especificar la ubicación y descripción plena de un documento en la web, esto lo hace más útil para un usuario final, pues los datos pueden ser usados en diferentes formas. Lo contrario ocurre con los datos definidos en formatos HTML o PDF, ya que no permiten dar significado a cada registro. Otra ventaja de XML es su capacidad de ser programable en diferentes idiomas con simples códigos de traducción, por el contrario HTML es un lenguaje específico, con lo cual hacer este tipo de cambios es muy dificultoso y PDF al ser una imagen, presenta el mismo problema (Valderrama, 2002). 


\section{eXtensible Business Reporting Language (XBRL).}

XBRL es un lenguaje que ha surgido en el entorno financiero, el cual es utilizado para la presentación de informes contables y que permite la comunicación, de forma estandarizada, entre empresas u organismos (Valencia, 2011; p 17). XBRL es una adaptación XML dirigida a regular la transmisión de información contable, principalmente la contenida en los estados financieros elaborados por las empresas.

\section{Beneficios del uso de XBRL,}

Entre las principales ventajas derivadas del uso de este nuevo lenguaje, se pueden identificar las siguientes:

- Reducción de costos y flexibilidad en la captura de datos.

- Reutilización de los datos. Cualquier usuario puede tomar una taxonomía estándar 0 propia y extenderla 0 distribuirla para cumplir con sus requerimientos específicos.

- $\quad$ Flexibilidad de información

- Facilita la toma de decisiones

- Facilita los procesos de comparación de la información financiera y de negocios que actualmente son realizados manualmente

- Soporta estándares contables internacionales así como también otros idiomas diferentes al inglés.

- Aumenta la confiabilidad de los datos

- Construye sistemas con menos probabilidad de error. Se disminuye su costo de desarrollo, mantenimiento y formatos de salida

- Depura en origen la información, se evita el envío de correcciones que incluso puede prevenir el reenvío de información a los organismos de regulación.

- Aumenta la eficiencia, ya que al combinar taxonomías, documentos XML y herramientas automatizadas permite procesar informes de negocio en forma automática, evitando la realización de procedimientos manuales de verificación, reingreso y comparación de datos

- Las taxonomías actúan como diccionarios, ofreciendo una definición explicita para cada elemento de dato con el objetivo de asegurar una interpretación única. 


\section{Documentos que componen XBRL.}

\section{Especificación de XBRL}

La especificación XBRL se denomina la piedra angular de este lenguaje. Es un documento maestro con carácter normativo que contiene una serie de estructuras y reglas generales a las que han de ajustarse todos los ficheros XML que se utilicen para representar la información contable a través de XBRL. Suministra una explicación técnica de lo que es XBRL y cómo trabaja. Define su estructura y también explica en detalle la sintaxis y semántica de las taxonomías y documentos de instancia de XBRL. Se puede decir que es un marco de referencia al cual se tienen que adaptar todos los trabajos de estandarización que se realicen.

\section{Taxonomía XBRL.}

Una taxonomía es un documento que define los elementos claves (numéricos o de texto) que deben ser incluidos en un documento de instancia, con el fin de elaborar un tipo particular de reporte financiero. En palabras simples, es una lista de todas las definiciones establecidas por las partes interesadas, muy parecido a lo que podría ser un plan de cuentas detallado para el reporte de información financiera, donde se define cada elemento de los Estados Financieros.

\section{Estructura de una Taxonomía.}

Una taxonomía XBRL está compuesta por un XML schema (o taxonomy schema) $y$ todos los linkbases contenidos en ese esquema 0 directamente referenciados en él. Puede estar definida en uno 0 varios archivos que pueden descomponerse en dos partes: conceptos y relaciones (Rial, 2008)

Los archivos que componen una taxonomía XBRL son los siguientes:

- Esquema de la taxonomía. En él se define el diccionario de términos a utilizar en la comunicación.

- Linkbase de etiquetas. Las etiquetas o textos asociados a los elementos del diccionario que pueden utilizarse en distintos idiomas y con distintos propósitos a la hora de construir representaciones de los informes.

- Linkbase de referencias. Las referencias a textos legales 0 normativas que fundamentan la base legal del concepto a modelar.

- Linkbase de presentación. Las reglas para construir una representación del informe que se pretende modelar.

- Linkbase de cálculo. Las reglas de cálculo entre elementos de la taxonomía que permiten validar los informes XBRL.

- Linkbase de definición. Reglas adicionales que permiten documentar relaciones entre elementos de la taxonomía y que se utilizarán para validar Ios informes. (Libro Blanco, XBRL, 2006). 


\section{XBRL en el intercambio de Informacion Financiera.}

En la cadena de suministro de información finaciera que se muestra en la figura, el $X B R L$ permite que los datos sean verificados, analizados y manipulados de acuerdo a la necesidad de los distintos participantes, todo ello sin modificar su integridad 0 interpretacion, ya que como se ha mencionado anteriormente, es XBRL extensible. (Rial, 2008)

Figura $N^{0} 1$ : XBRL y sus aportes al intercambio de información financiera

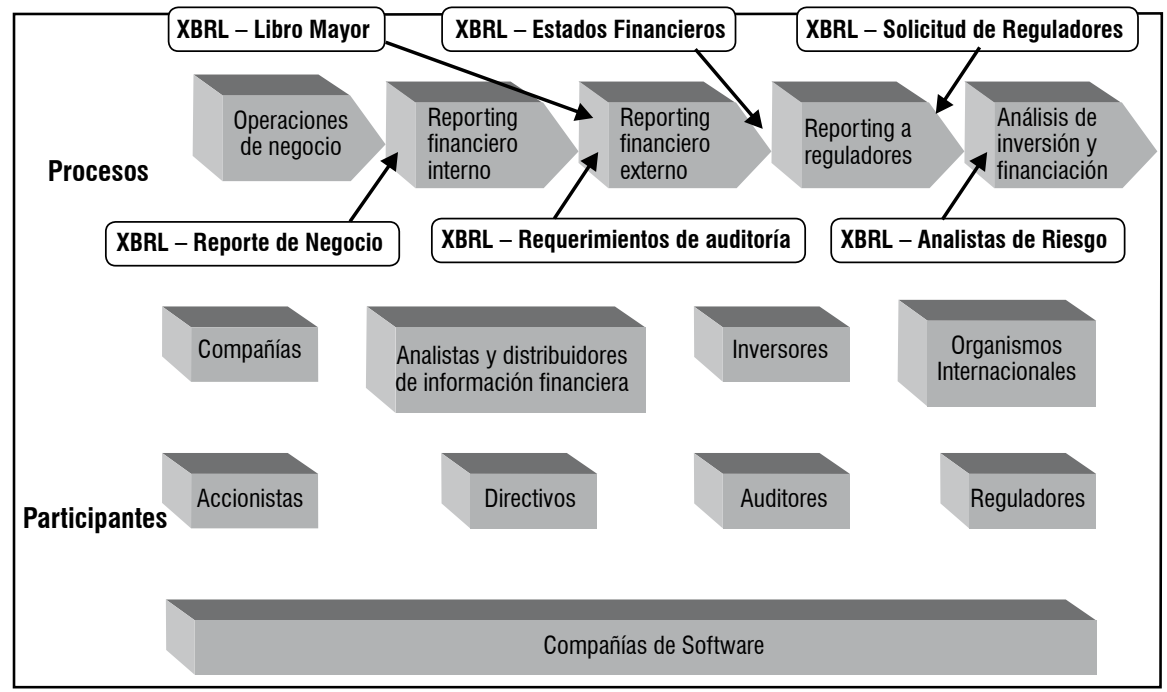

Fuente: Superintendencia de Valores y Seguros (SVS), 2013

La Superintendencia de Valores y Seguros (SVS) es una institución autónoma, que se relaciona con el Gobierno a través del Ministerio de Hacienda. Tiene por objeto la superior fiscalización de las actividades y entidades que participan de los mercados de valores y de seguros en Chile. Así, a la SVS le corresponde velar porque las personas o instituciones supervisadas, desde su inicio hasta el término de su liquidación, cumplan con las leyes, reglamentos, estatutos y otras disposiciones que rijan el funcionamiento de estos mercados. (Superintendecia de Valores y Seguros, 2013).

\section{Entidades Fiscalizadas.}

Entidades del Mercado de Valores: Agentes de Valores, Bolsas de Valores, Clasificadoras de Riesgo, Corredores de Bolsa, Empresas de Depósito de Valores, Empresas de Auditoría Externa, Fondo de Inversión de Capital Extranjero (FICE), Fondos de Inversión Fondos Mutuos, Fondos para la Vivienda, Sociedades Administradoras de Fondos de Inversión de Capital Extranjero, Sociedades 
Administradoras de Fondos de Inversión, Sociedades Administradoras de Fondos Mutuos, Sociedades Administradoras de Fondos para la Vivienda, Sociedades Administradoras Generales de Fondos, Sociedades anónimas abiertas y emisores de valores, entre otros.

Entidades del Mercado de Seguros: Compañías de Seguros de Vida, Compañías de Seguros Generales, Compañías de Seguros de Crédito, Corredores de Seguros, Asesores previsionales, Clasificadoras de Riesgo, Empresas de Auditoría Externa, entre otras.

Otras Entidades: abogados calificadores, Cuerpo de Bomberos, Empresas públicas, Entidades Informantes, Fondos Solidarios de Crédito Universitario, Organizaciones Deportivas Profesionales, Sociedades afectas al impuesto específico a la actividad minera, entre otras.

\section{Taxonomía XBRL SVS CL-CI}

La Taxonomía XBRL SVS CL-Cl representa las normas (IFRSs1 y PCGA chilenos) aplicables en el momento de su publicación. Se compone de un único archivo de esquema (schema) que importa los elementos de IFRS-GP y define conceptos adicionales.

\section{Formato de la Taxonomía}

A continuación se realizara una explicación del formato y el contenido de la Taxonomía CL-Cl.

\begin{tabular}{|l|l|l|l|}
\hline \multicolumn{2}{|l|}{$[110000]$ Información general sobre estado financieros } & Tipo & Referencias \\
\hline label & texto & IAS 1 Structure and content \\
\hline \multicolumn{2}{|l|}{ Información a revelar sobre información general sobre los estado financieros } & texto & IAS 1.51 a \\
\hline & Nombre de la entidad que informa u otras formas de identificación & texto & \\
\hline & Rut de la entidad que informa & texto & \\
\hline & Número del registro de valores &
\end{tabular}

Fuente: (SVS, Taxonomía llustrada)

\section{Primera columna - jerarquía.}

La primera columna de la imagen indica la jerarquía de las Normas (IFRS y de Chile):

- El encabezado de columna representa el nombre de un componente de la Norma. Cada título de la columna va precedido de un número de seis cifras entre corchetes, con un valor entre [100000] y [999999]. Estos números son artificiales, no están relacionados con la norma y proporcionan la funcionalidad de visualización y de clasificación.

- Las filas por debajo de los encabezados de columna representan los 
elementos que pertenecen a este componente, los cuales son requisitos de revelación de las Normas.

\section{Segunda columna - formato}

- Bloque de texto - indica que el formato de revelación es un bloque de texto en formato HTML.

- Texto - indica que el formato de revelación es texto sin formato.

- $\quad$ aaaa-mm-dd - indica que el formato de la revelación es fecha.

- $\quad \mathrm{X}$ - indica que el formato de la revelación es un valor monetario.

- $(X)$ - indica que el formato de la revelación es un valor monetario que se presenta como negativo.

- $\quad X . X X$ - indica que el formato de la revelación es un valor decimal.

- Acciones - indica que el formato de la revelación es un número de acciones.

- _ _ _ Indica que el formato de la revelación es la suma total de las filas anteriores.

- Tabla - indica el comienzo de una revelación de dos dimensiones

- $\quad$ Eje - indica un eje en una revelación dimensional.

- Miembro - indica un miembro de un eje.

- $\quad$ Línea de partidas - indica el comienzo de una serie de revelaciones para una tabla.

- Una celda en blanco indica que una revelación no es necesaria.

\section{Tercera columna - referencias}

La tercera columna indica la sección/párrafo de la Norma para una revelación dada.

\section{Soporte para XBRL en Chile.}

La SVS, a través de su página web publica un listado de empresas que ofrecen soluciones y soporte al estándar XBRL. A su vez menciona que la SVS no revisa los productos que ofrecen estas empresas, ni otorga certificados de calidad o garantía sobre el buen funcionamiento del software XBRL. 
A continuación se presenta el listado de proveedores antes mencionado:

\begin{tabular}{|l|l|}
\hline Proveedores & Sitio Web \\
\hline ASICOM (Representante de Clarity) & http://www.asicom.com/ \\
\hline BR-AG & http://www.br-ag.eu/ \\
\hline DBNET & http://www.dbnet.cl/ \\
\hline DSIC & http://www.dsic.cl/ \\
\hline DyT SYSTEMS & $\underline{\text { http://www.dytsystems.cl/ }}$ \\
\hline PAPERLESS & $\underline{\text { http://www.paperlessla.com/ }}$ \\
\hline PERFEKTIO (Representante de "Edicom") & $\underline{\text { http://www.perfektio.cl/ }}$ \\
\hline PRICEWATERHOUSECOOPERS CHILE & $\underline{\text { http://www.pwc.com/cl/es/XBRL/index.jhtml }}$ \\
\hline PRISMA CONSULTING (representante de Altova) & $\underline{\text { http://www.prismaconsulting.cl/ }}$ \\
\hline REPORTING STANDARD S.L & $\underline{\text { http://www.reportingstandard.com/ }}$ \\
\hline SAP & $\underline{\text { http://www.sap.com }}$ \\
\hline TECNOLOGÍA, DESARROLLO \& NEGOCIOS S.A & $\underline{\text { http://www.xbrl-ifrs.cl/ }}$ \\
\hline
\end{tabular}

Fuente: Superintendencia de Valores y Seguros (2013).

\section{Proceso de Convergencia a Estándares Internacionales de Contabilidad}

\section{Normas Internacionales de Contabilidad.}

Las Normas Internacionales de Contabilidad Financiera (IFRS) son emitidas por el International Accounting Standards Board (IASB) desde el año 2000 con el objetivo de desarrollar un estándar de información comparable, transparente y de alta calidad en los estados financieros y en otros tipos de información financiera, que facilite las decisiones económicas. Estas normas están diseñadas para ser aplicadas en la información financiera de todas las entidades con fines de lucro, incluyendo las empresas públicas. A nivel mundial, cada vez son más países los que están adoptando o convergiendo a las IFRS. Por su parte, el IASB efectúa una permanente revisión y ajuste de las normas y criterios aplicables. 


\section{Proyecto de Convergencia a IFRS en Chile.}

El proceso de convergencia a IFRS se detalla en la siguiente imagen:

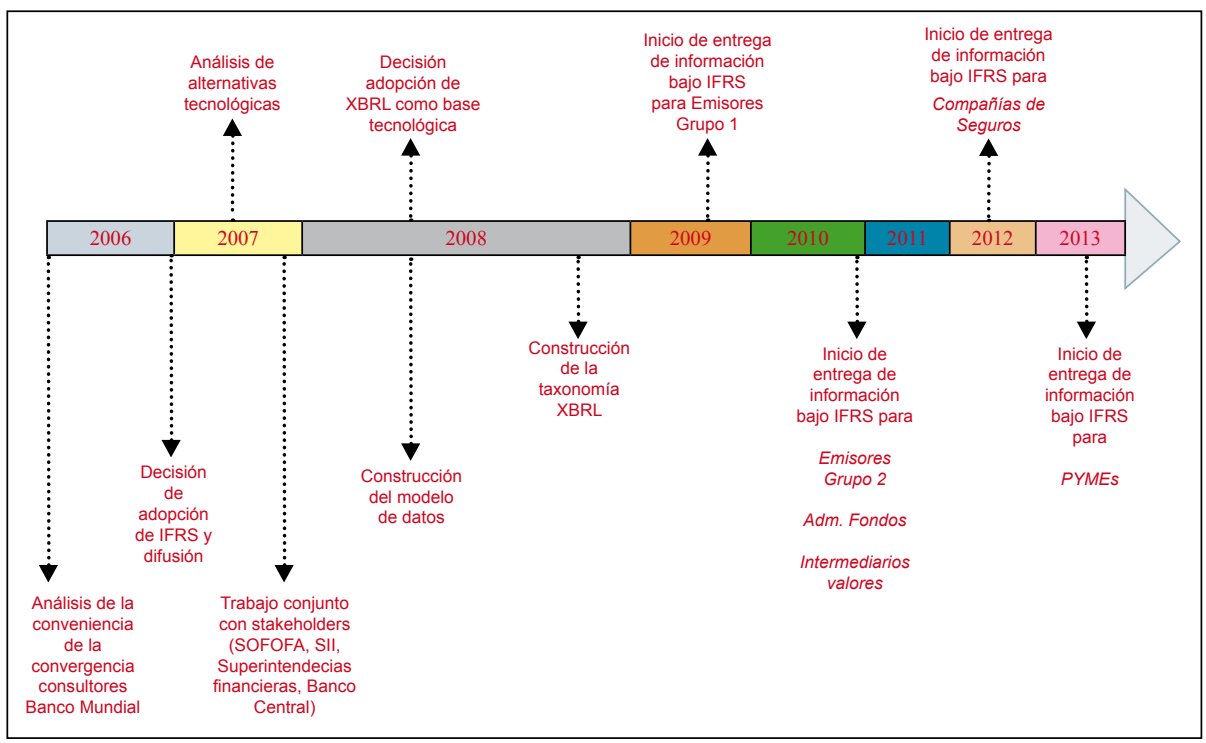

Fuente: Coloma (2011).

\section{Propuesta Metodológica.}

La investigación tiene un enfoque cualitativo con un alcance de comprensión. Se realizan entrevistas a participantes de un conjunto de empresas, Sociedades Anónimas Abiertas u otras, que sean supervisadas por la SVS y que estén en proceso de implementación 0 utilicen actualmente el estándar XBRL para la elaboración de sus Informes Financieros. 
La información se procesará por medio de las siguientes categorías de análisis.

\begin{tabular}{|l|l|}
\hline $\begin{array}{l}\text { CATEGORIA 1 } \\
\text { Uso de las TIC }\end{array}$ & $\begin{array}{l}\text { Opinión sobre el uso de las tecnologías de información } \mathbf{y} \\
\text { comunicación en la organización. }\end{array}$ \\
\hline $\begin{array}{l}\text { CATEGORIA 2 } \\
\text { Información Financiera }\end{array}$ & Oportunidad de la información financiera para la toma de decisiones. \\
\hline $\begin{array}{l}\text { CATEGORIA 3 } \\
\text { XBRL / IFRS }\end{array}$ & $\begin{array}{l}\text { Uso del XBRL para facilitar la implementación de las Normas } \\
\text { Internacionales de Contabilidad Financiera (IFRS) }\end{array}$ \\
\hline $\begin{array}{l}\text { CATEGORIA 4 } \\
\text { Estructura interna }\end{array}$ & $\begin{array}{l}\text { Cambios en la estructura interna de la organización a causa del } \\
\text { requerimiento de la SVS. }\end{array}$ \\
\hline $\begin{array}{l}\text { CATEGORIA 5 } \\
\text { Capacitación }\end{array}$ & $\begin{array}{l}\text { Capacitación a los usuarios encargados de preparar los reportes } \\
\text { mediante XBRL }\end{array}$ \\
\hline $\begin{array}{l}\text { CATEGORIA 6 } \\
\text { Proveedores }\end{array}$ & Opinión sobre los proveedores del software XBRL \\
\hline
\end{tabular}

\section{Resultados, discusión y conclusiones.}

De acuerdo a las entrevistas realizadas, se obtiene la información a analizar, como lo establecen previamente las categorías de análisis en la metodología de investigación.

\section{Conclusiones del análisis de resultados.}

\begin{tabular}{|c|c|}
\hline Uso de las TIC & $\begin{array}{l}\text { Las Tecnologías de Información y Comunicación son un elemento importante } \\
\text { dentro de la organización, sobre todo en aquellas donde se trabaja con un } \\
\text { alto volumen de transacciones. Permiten generar eficiencia en los procesos y } \\
\text { analizar la información que se requiere para la toma de decisiones en forma } \\
\text { oportuna. } \\
\text { Otros aspectos importantes son la incorporación de controles sobre dichas } \\
\text { tecnologías y lograr una coordinación entre los sistemas y las personas que } \\
\text { trabajan con ellos. }\end{array}$ \\
\hline $\begin{array}{l}\text { Información } \\
\text { Financiera }\end{array}$ & $\begin{array}{l}\text { Actualmente la elaboración del XBRL se realiza para cumplir con las } \\
\text { exigencias del Regulador. Las empresas no utilizan la información financiera, } \\
\text { contenida en este reporte, para realizar algún tipo de análisis, consideran que } \\
\text { su elaboración genera un costo adicional que no aporta un mayor beneficio. }\end{array}$ \\
\hline XBRL/IFRS & $\begin{array}{l}\text { Los aspectos positivos de este nuevo reporte, que se comienza a exigir a } \\
\text { causa del proceso de convergencia a Normas Internacionales de Información } \\
\text { Financiera son, que debería proporcionar una mejor visualización de los } \\
\text { Estados Financieros y que al validar la información financiera contenida, } \\
\text { proporciona mayor seguridad para la gerencia, accionistas y otros usuarios } \\
\text { de dicha información. También se destaca el uso de una taxonomía que } \\
\text { contribuye a estandarizar la información financiera. }\end{array}$ \\
\hline $\begin{array}{l}\text { Cambios en la } \\
\text { estructura interna }\end{array}$ & $\begin{array}{l}\text { No se han generado cambios importantes en la estructura interna de las } \\
\text { organizaciones a causa de este requerimiento. } \\
\text { La labor de prepararel archivoXBRL ha sido encomendada alos departamentos } \\
\text { encargados de confeccionar los Estados Financieros, aumentando la carga } \\
\text { laboral de los trabajadores, quienes reconocen que la preparación de este } \\
\text { archivo demanda mucho tiempo, sobre todo en sus inicios. }\end{array}$ \\
\hline
\end{tabular}




\begin{tabular}{|l|l|}
\hline \multirow{5}{*}{ Capacitación } & $\begin{array}{l}\text { El proveedor en principio es quien realiza una inducción sobre el manejo del } \\
\text { software y mantiene informados, a los encargados de preparar el archivo } \\
\text { XBRL, sobre nuevas actualizaciones de las Taxonomías. } \\
\text { Se plantea que debiese existir mayor capacitación para los usuarios de la } \\
\text { información financiera en general, ya sea el Regulador, analista de inversiones, } \\
\text { analistas de bancos, etc. }\end{array}$ \\
\hline Proveedores & $\begin{array}{l}\text { En general la opinión sobre los proveedores del software es buena, ya que } \\
\text { en ciertas situaciones donde se han generado problema, han respondido en } \\
\text { forma oportuna y han proporcionado apoyo a los encargados de elaborar el } \\
\text { archivo XBRL. } \\
\text { En cuanto a su elección, se han utilizado criterios como: ver el proveedor que } \\
\text { más reporta o ver el que utiliza la competencia. }\end{array}$ \\
\hline
\end{tabular}

\section{Discusión.}

La importancia del uso de las Tecnologías de Información y Comunicación (TIC) en las organizaciones es corroborada tanto por la teoría como por los entrevistados. Al respecto Illanes (1999) sostiene que la tecnología es puesta al servicio de la información como un medio para favorecer las decisiones y Ganga \& Águila, (2006) menciona que las tecnologías de la información han sido introducidas en las empresas para automatizar la coordinación y la gestión de la información.

Por su parte los entrevistados, según su experiencia, afirman que las TIC son de vital importancia para las organizaciones, tanto así que algunos las han definido como la columna vertebral de toda organización, esto debido al gran volumen de transacciones e información que manejan las compañías. Una opinión sostiene que la única forma de generar eficiencia, ahorrar costos y efectuar los análisis a tiempo es mediante el uso de las tecnologías de información, pero a su vez hace hincapié en que por sí sola la tecnología no es buena, sino que se requiere de la incorporación de controles para disminuir los riesgos asociados a esta.

Bastias (2009) señala que los sistemas de información están compuestos tanto por TI (tecnología de información) como también por usuarios, aquellos que están a cargo de la TI y aquellos que utilizan la información que provee el sistema. Dicha teoría se relaciona con lo expuesto por uno de los entrevistados, la que señala que debe existir coordinación entre las tecnologías de información, los procesos y las personas que trabajan con ella. De ser así, esto se vuelve fundamental y contribuye a la organización y a sus procesos de negocio.

Con respecto a la importancia de la información financiera, el Marco Conceptual, elaborado para la preparación y presentación de los Estados Financieros, establece que para ser útil, la información debe poseer ciertas características cualitativas que son: comprensibilidad, relevancia, fiabilidad y comparabilidad. Los entrevistados se refieren a esto mencionando que la información que contiene el reporte XBRL cumple con las características de: fiabilidad, ya que al contener un validador que no 
permite la publicación de información errónea, los usuarios pueden tener certeza de que los Estados Financieros representan la imagen fiel de la organización; y comparabilidad, ya que al basarse en una Taxonomía, permite la comparación de los Estados Financieros de cualquier empresa, ya sea nacional o internacional.

Otros entrevistados plantean una opinión crítica con respecto a este tópico, mencionan que en teoría el XBRL contribuye a obtener Información Financiera de mayor calidad, permitiendo a sus usuarios exportar datos y hacer comparativos para mejorar la toma de decisiones, sin embargo en la práctica esto no ocurre en Chile, ya que aún el XBRL se encuentra en una etapa inicial, donde no se aprovechan todos sus beneficios, elaborándolo solo para cumplir con el requerimiento del ente regulador.

En Chile el XBRL fue elegido como base tecnológica para estandarizar la información, producto de la adopción de las Normas Internacionales de Información Financiera. La Superintendencia de Valores y Seguros menciona que algunos de los beneficios de adoptar IFRS son: aumentar la calidad y comparabilidad de la información financiera, lograr un estándar único que reduce los costos de elaboración de información para empresas que operan a nivel internacional y cotizan en otros mercados, tener un mejor acceso y capacidad de las empresas para obtener financiamiento en otras plazas y la reducción de costos de procesamiento de información para los inversionistas (por comparabilidad). Los entrevistados al referirse a este tema plantean una opinión crítica con respecto al trabajo del Regulador, según su experiencia hasta ahora, la elaboración del XBRL se realiza solo para cumplir con una exigencia, ya que si bien en teoría reconocen los beneficios del XBRL, en la práctica no se utiliza para realizar análisis o comparativos que ayuden a la toma de decisiones por parte de los usuarios.

A partir de la exigencia impuesta por la Superintendencia de Valores y Seguros, las empresas Sociedades Anónimas Abiertas, han tenido que incorporar dentro de sus procesos la elaboración del Reporte XBRL. Los entrevistados mencionan que las empresas han tenido que asumir un costo, al contratar los servicios de un proveedor para realizar la conversión del archivo, que no genera mayor beneficio. 


\section{Conclusiones.}

Al finalizar el presente estudio sobre la experiencia de las organizaciones en cuanto al uso e implementación del XBRL, a causa de la exigencia impuesta por la Superintendencia de Valores y Seguros, es posible concluir:

La decisión de las autoridades de converger a Normas Internacionales de Información Financiera (IFRS) sin duda ha generado cambios importantes al interior de las organizaciones. Uno de ellos es la obligación impuesta por el regulador de reportar bajo XBRL, estándar elegido como base tecnológica para la estandarización de la información financiera.

Teóricamente el XBRL aporta grandes beneficios a las organizaciones y a sus procesos de negocios. Principalmente se destaca la reducción de costos, flexibilidad de la información, soporte de estándares contables internacionales y aumento de la confiabilidad de los datos, sin embargo la experiencia de las organizaciones objeto de estudio no ha sido muy alentadora.

Para la elaboración del reporte XBRL, las empresas han asumido un costo relacionado con la conversión del archivo. También ha generado una sobre carga laboral en los encargados de su preparación, quienes reconocen que es un proceso tedioso, porque básicamente consiste en la re digitación de la información contenida en el informe FECU, cuyo reporte sigue siendo obligatorio. Por otra parte el XBRL ha sido diseñado para facilitar la transmisión de la información financiera y ayudar a los distintos usuarios en los procesos de toma de decisiones, lo cual no ocurre, ya que para esto las organizaciones prefieren utilizar sus reportes internos.

En base a los antecedentes anteriormente expuestos y a la información recopilada durante la investigación, surge la interrogante sobre si es necesario para las organizaciones confeccionar sus Estados Financieros bajo este estándar. Actualmente el reporte de XBRL se realiza solo para cumplir con una exigencia y no existe mayor interés en aprovechar los beneficios que se derivan de la estandarización de la información.

En cuanto a la labor de la Superintendencia, esta es cuestionada por su falta de compromiso y escasa preocupación por las necesidades de las organizaciones obligadas a reportar. Se cree que con esta medida el ente regulador busca cumplir con estándares internacionales más que generar un beneficio en el proceso de intercambio de la información financiera.

También se cuestiona la falta de coordinación entre el equipo encargado de la revisión de IFRS y el equipo encargado de la revisión del archivo XBRL, ambos no se relacionan suficientemente entre sí, produciéndose desacuerdos en la aprobación de los informes. 
En cuanto a los plazos de revisión, estos se consideran extensos, pudiendo transcurrir aproximadamente seis meses. Si un usuario quisiera utilizar el archivo XBRL para distintos análisis, puede hacerlo accediendo al sitio web de la Superintendencia, sin embargo no se consideraría información fiable, ya que se menciona que el archivo XBRL está bajo revisión, por lo cual dicho usuario preferiría optar por descargar el informe FECU elaborado en formato PDF.

A cinco años de la decisión de adoptar el estándar internacional XBRL, se reconoce que el proceso de implementación aún se encuentra en una etapa inicial. La resistencia al cambio por parte de las organizaciones y la poca información que existe sobre este tema son factores determinantes al momento de evaluar el éxito de esta medida impuesta por la Superintendencia. Es preciso que los distintos usuarios que participan en la cadena de intercambio de la información financiera, se capaciten e interioricen con respecto a los beneficios que se pueden obtener al reportar bajo este estándar.

Si bien esta obligación no ha generado cambios significativos al interior de las organizaciones, la percepción que los entrevistados tienen con respecto a este tema es buena a largo plazo, por lo cual se espera que en el futuro se logre masificar el uso de XBRL para el reporte de información financiera y de negocios.

Finalmente con respecto al uso de la tecnologías de información y comunicación, su uso e incorporación se considera importante debido al gran volumen de transacciones que manejan las organizaciones. Las TIC aportan eficiencia en todos los procesos de negocio, sin embargo se considera imprescindible que su uso este acompañado de la incorporación de controles adecuados y que exista plena coordinación entre los usuarios y dichas tecnologías. 


\section{Bibliografía.}

- Andreu, R., Ricarte, J., \& Valor, J. (1991). Estrategía y Sistemas de Información. Madrid: Mc Graw-Hill.

- Bastias, Jeniffer (2009). Estudio de los sistemas de información informáticos y de las tecnologías de la información, desde la óptica del SIC. Universidad de Valparaíso, Chile.

- Biblioteca del Congreso Nacional de Chile. (13 de diciembre de 2005). La Globalización y sus efectos. Chile. Recuperado el 15 de mayo de 2013, de http://www.bcn.cl/carpeta temas/temas portada.2005-11-29.5590492629

- Cañibano, L. (2004). Información Financiera y gobierno de la empresa. Revista internacional Legis de contabilidad \& auditoría , 158-235.

- Coloma C. (2011) Proceso de convergencia a estándares internacionales de Contabilidad IFRS (NIIC) e IPSAS (NICSP) en Seminario sobre Normas Internacionales de Contabilidad para Sector Público en Santiago Chile, Mayo 2011 (pág. 4).

- Consejo Nacional del Colegio de Contadores de Chile A. G. (2006). Marco Conceptual para la Preparacion y Presentacion de Estados Financieros. Chile.

- Libro Blanco 4 XBRL concepto y recomendaciones (2006). XBRL España, de http://www.xbrl.es/downloads/libros/Libro Blanco.pdf

- Lorca, P., Suarez, J. d., \& García, J. (2004). La comunicación de la información: El papel del XBRL. Recuperado el 15 de julio de 2013, de http://www.asepelt.org/ficheros/File/Anales/2003\%20-20Almeria/ asepeltPDF/102.PDF

- Montes, C., Montilla, 0. d., \& Mejía, E. (2006). Análisis del marco conceptual para la preparación y presentación de estados financieros conforme al modelo internacional iasb. Estudios Gerenciales , 61-83.

- Morilla, P. (2007) El estándar XBRL y los Mercados de Valores: Primera Edición: Aspectos Tecnológicos. [Versión electrónica] Chile. Disponible en versión electrónica en http://www.xbrl.es/downloads/ ContenidoCurso0nlineCEDDET1Ed.pdf

- Real Academia Española. (2013). Obtenido de http://lema.rae.es/ drae/?val=globalizacion. 
- Rial, C. (2008). XBRL y sus aportes al intercambio de información financiera. Uruguay.

- Superintendecia de Valores y Seguros (SVS) Recuperado el 20 de agosto de 2013, de www.svs.cl

- Taxonomía llustrada SVS CL-Cl (2013) de, http://www.svs.cl/sitio/xbrl/ html/tax2013.php

- Valderrama, M. (2002). XBRL: Tecnología contable para el reporte digital, de http://www.javeriana.edu.co/fcea/cuadernos contab/vol5 n 20/ vol5 20_1.pdf

- Valencia, J. (2011). Modelo de Meta-metadatos XBRL. Leganes: Universidad Carlos III de Madrid.

- Zuñiga, J. (2007). Diagnóstico actual sobre la percepción de los estándares XML y XBRL en el campo laboral de auditores externos en empresas auditoras de Santiago de Chile. Santiago: Universidad Santiago de Chile. 